Protection of bronze artefacts through polymeric coatings based on nanocarriers filled with corrosion inhibitors

Martina Salzano de Luna, Giovanna Buonocore, Gabriella Di Carlo, Chiara Giuliani, Gabriel M. Ingo, and Marino Lavorgna

Citation: 1736, 020048 (2016); doi: 10.1063/1.4949623

View online: http://dx.doi.org/10.1063/1.4949623

View Table of Contents: http://aip.scitation.org/toc/apc/1736/1

Published by the American Institute of Physics 


\title{
Protection of Bronze Artefacts Through Polymeric Coatings Based on Nanocarriers Filled with Corrosion Inhibitors
}

\author{
Martina Salzano de Luna ${ }^{\mathrm{a}}$, Giovanna Buonocore ${ }^{\mathrm{a}}$, Gabriella Di Carlo ${ }^{\mathrm{b}}$, Chiara \\ Giuliani $^{\mathrm{b}}$, Gabriel M. Ingo ${ }^{\mathrm{b}}$ and Marino Lavorgna ${ }^{\mathrm{a}}$ \\ ${ }^{a}$ Institute of Composite and Biomedical Materials, National Research Council (IPCB-CNR), \\ Piazzale Fermi 1, 80055 Portici (NA), Italy \\ ${ }^{b}$ Institute for the Study of Nanostructured Materials, National Research Council (ISMN-CNR), \\ Via Salaria km 29,3, 00015 Monterotondo (RM), Italy
}

\begin{abstract}
Protective coatings based on polymers synthesized from renewable sources (chitosan or an amorphous vinyl alcohol based polymer) have been prepared for the protection of bronze artifacts from corrosion. Besides acting as an effective barrier against corrosive species present in the environment, the efficiency of the coatings has been improved by adding corrosion inhibitor compounds (benzotriazole or mercaptobenzothiazole) to the formulations. The liquid medium of the formulations has been carefully selected looking at maximizing the wettability on the bronze substrate and optimizing the solvent evaporation rate. The minimum amount of inhibitor compounds has been optimized by performing accelerated corrosion tests on coated bronze substrates. The inhibitors have been directly dissolved in the coating-forming solutions and/or introduced by means of nanocarriers, which allow to control the release kinetics. The free dissolved inhibitor molecules immediately provide a sufficient protection against corrosion. On the other hand, the inhibitor molecules contained in the nanocarriers serve as long-term reservoir, which can be activated by external corrosion-related stimuli in case of particularly severe conditions. Particular attention has been paid to other features which affect the coating performances. Specifically, the adhesion of the protective polymer layer to the bronze substrate has been assessed, as well as its permeability properties and transparency, the latter being a fundamental feature of protective coating for cultural heritages. Finally, the protective efficiency of the produced smart coatings has been assessed through accelerated corrosion tests.
\end{abstract}

Keywords: Polymeric coating; Nanocarrier; Corrosion protection; Cultural heritages

PACS: $81.15 .-\mathrm{z} ;$ 82.35.Np; 82.35.Gh; 68.60.-p

\section{INTRODUCTION}

Contemporary art is a major challenge for conservators since there is a significant lack of established conservation methodologies that can safely tackle the degradation of materials used by contemporary artists. Up to now the most widespread approach for corrosion protection of metallic artefacts is the application of protective polymer coatings, which act as an effective barrier against corrosive species present in the environment. However, natural polymer aging and external factors may lead to the formation of micro-pores and micro-cracks which interrupt the barrier effect and allow the direct ingress of corrosive species into the metal surface. Extra functionalities have thus to be imparted to the polymeric coatings in order to provide long-term protection to the substrates. In this sense, direct incorporation of corrosion inhibitors into coating formulations, in order to confer active protection when the coating barrier properties fail, could represent a viable solution to the problem. However, detrimental interactions may occur between the active species and the matrix within which they are dispersed, such as the loss of the inhibition capability, coating degradation or both [1]. A clever and promising strategy to overcome such hurdles is represented by the encapsulation of the inhibitors within inert host nanostructures, generally referred as nanocarriers. The immediate advantage offered by the latter is the possibility of controlling the release of the active compound. Actually, once the corrosion process is started, such nanostructures allows a controllable leaching of the inhibitor triggered by a corrosion related stimuli $[2,3]$. When dealing with cultural heritages, however, further severe and restrictive characteristics of the protective layer are needed. Specifically, besides the corrosion protection, 
the coating have to be optically transparent in order to preserve the appearance of the artifact, and it has to be easily removable without compromising the integrity of the metallic surface. As a result, the production of effective and reliable protective coatings suitable for metallic artifacts is still in its early stages and it represents an imperative and yet demanding task for material scientists. The present research activity perfectly fits in this frame, its aim being the development of smart coating formulations for the protection of $\mathrm{Cu}$-based alloy (bronze) artworks stored or exhibited in indoor environment.

\section{EXPERIMENTAL}

The protective coatings are based on polymers synthesized from renewable sources: chitosan (CS) and an amorphous vinyl alcohol based polymer (Nichigo G-Polymer, GP , by Nippon Gohsei, Japan). Two kinds of nanoparticles have been exploited as nanocarriers: halloysite (HNTs) and layered double hydroxides (LDHs). HNTs have a typical cylindrical shape, whose open edges and inner lumen allow the encapsulation of the inhibitor compound. Differently, LDHs consist of stacks of positively charged layers between which the inhibitor compound can be intercalated. The loading process has been carried out by an anion-exchange reaction. Benzotriazole (BTA) and mercaptobenzothiazole (MBT) have been used as active compound since their anticorrosion efficiency has been already recognized for different metal substrates. The coating formulations have been prepared in the form of liquid mixtures and have been applied on the bronze substrates ( $\mathrm{Cu} 85 \%$, Sn $5 \%$, Zn 5\%, Pb $5 \%$ ) by either manual deposition or spray coating. The alloy composition was selected based on the most common metal composition of bronze works of art [4]. Contact angle measurements have been exploited to assess the wetting behavior of the coating formulations on the bronze disks. To assess the efficacy of the protective polymer layers, accelerated corrosion tests have been performed on the coated bronze substrates. The surface properties of bare $\mathrm{Cu}$-based alloy with and without polymer protective coatings have been investigated by scanning electron microscopy (SEM).

\section{RESULTS AND DISCUSSION}

The research activity has been basically divided into three front of research. The first part concerns the loading of the corrosion inhibitor into the nanoparticles and the characterization of the obtained nanocarriers. The second part regards the optimization of the coating formulation, mixing protocol and deposition procedure. Finally, the investigation of the performances of the protective coatings represents the third front of research.

\section{Loading Efficiency and Kinetics of Release}

Besides assessing the amount of inhibitor encapsulated in the nanocarriers by thermogravimetric analyses, the release kinetics of the active specie has been also monitored by UV-vis spectroscopy in various conditions, such as different concentration of chloride species and different $\mathrm{pH}$. Due to intrinsic differences in the structure and morphology of the pristine nanoparticles, the amount of encapsulated inhibitor, as well as the release kinetics, are different for the two kinds of nanocarriers. Actually, such differences reflect the different procedures adopted for the loading process and also the mechanisms by which the inhibitor is released. Specifically, for the HNTs the release is simply related to the leakage of the compound from the inner lumen. The process thus cannot be controlled unless by using targeted expedients, such as stopper networks for the tube edges. On the contrary, in the case of LDH nanoparticles, the release of active compound is triggered by anion-exchange phenomena in which corrosion aggressive chloride ions are involved.

\section{Optimization of the Coating Formulations}

The optimization of the coating formulation represents the core of the performed activity and essentially concerns the selection of the liquid phase, the determination of the inhibitor amount which is effective against corrosion and the optimization of the final composition of the formulations. The liquid medium of the formulation represent a key factor as the adhesion between the coating and the artefact surface is decisive to provide a long-term protective action. Moreover, a good affinity between the coating-forming formulation and the metallic substrate is essential to facilitate the deposition process and ensure a smooth surface appearance of the resulting polymer layer. In this perspective, a water/ethanol (W/E) 50/50 ( $\mathrm{vol} / \mathrm{vol})$ mixture has been selected as liquid phase because it guarantees the best wettability on the bronze substrates (Figure 1.a), yet matching the solubility range of the investigated polymers. Actually, an acid solvent is needed for the complete dissolution of CS, and typically acetic 
acid (AA) is used for such a purpose. The influence on the wetting behavior of the addition of AA to the W/E mixture is negligible (Figure 1.b). However, it negatively affect the appearance of the substrate due to the interaction of the AA with the $\mathrm{Pb}$ present in the substrates. Such a difficulty has been overcome by using D-(+)-gluconic acid $\delta$ lactone: the wettability is preserved and it does not interact with the bronze disks (Figure 1.b).
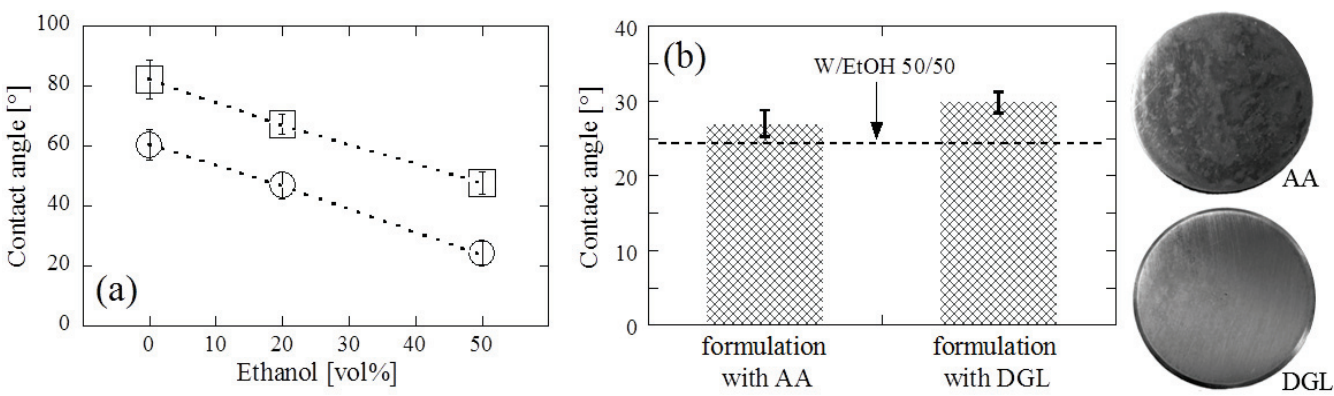

FIGURE 1. (a) Contact angle of water/ethanol mixtures on bronze substrate as a function of ethanol content. Data for substrates tested soon after (circles) and 3 days after (squares) lapping. (b) Effect on the formulation wettability and coating appearance of the addition of AA (picture on top) and DGL (picture on bottom) to the chitosan-based coatings.

Concerning the GP-based coatings, further expedients have been adopted. Specifically, the produced coatings are not homogenous and opaque, suggesting that GP possess a scarce affinity with the bronze substrate. To improve the latter, GP has been mixed with small amounts of polyallylamine (PAA). Actually, the miscibility between GP and PAA guarantee the preservation of the coating transparency. Moreover, the amine groups of the PAA favors the interaction with the substrate. The tuning of the GP/PAA ratio has allowed the straightforward production of homogenous coatings (Figure 2). In addition, GP can be easily dissolved in water, even at room temperature. Consequently, a crosslinking step is needed to improve the coating water-resistance and confer high barrier properties. On the other hand, the chemical reactions occurring during this step have not to compromise the surface integrity and stability of the artefacts. Thus, the crosslinking agent and catalyzer have been properly selected and the evolution of the $\mathrm{pH}$ during the process have been monitored. Moreover, the extent of the crosslinking has been tuned in order to guarantee the ease of removal of the coating.
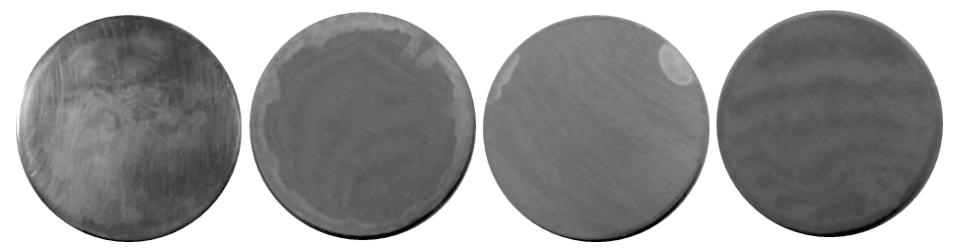

FIGURE 2. Pictures of bronze disks with coatings based on neat GP and GP/PAA blends at different compositions. From left to right: GP/PAA 100/0, 98/2, 95/5, 90/10 (wt/wt).

The composition of the coating-forming formulation has been identified looking at providing a sufficient amount of the anticorrosion compound and yet preserving the transparency and structural integrity of the coating. Moreover, due to the different loading capacity of the pristine nanoparticles, the possibility of synergistically using different kinds of nanocarriers to ensure an effective inhibitor concentration in the coating has been explored. Alternatively, the inhibitors have been introduced directly in the coating formulation and contextually also by addition of nanocarriers. In this case, the inhibitors freely distributed in the polymer matrix immediately provide the protection against corrosion. Instead, those contained in the nanocarriers serve as long-term reservoir, which can be activated by corrosion-related stimuli. Note that particular care has been paid to the dispersion step of the nanocarriers in the liquid medium, being the presence of micron-sized aggregates responsible for alteration of the transparency.

\section{Performances of the Protective Coatings}

To verify the applicability of the selected formulations, the analysis is essentially focused on the evaluation of the integrity and homogeneity of the applied coatings, its aesthetic appearance and ability to prevent/inhibit corrosion 
processes. The optical pictures an SEM micrographs of representative bare and CS-coated bronze substrates are shown in Figure 3 before and after accelerated corrosion tests.
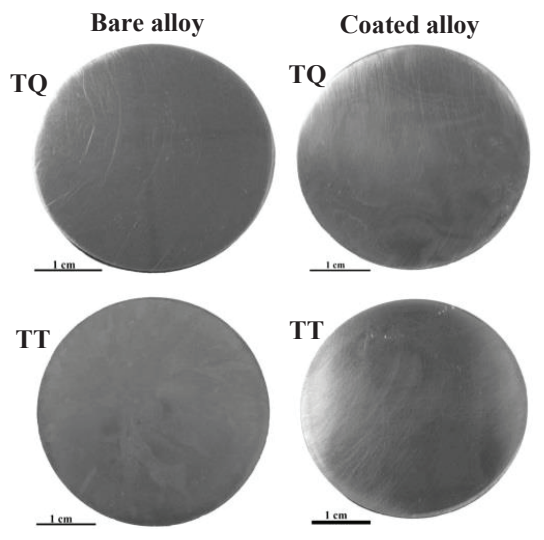

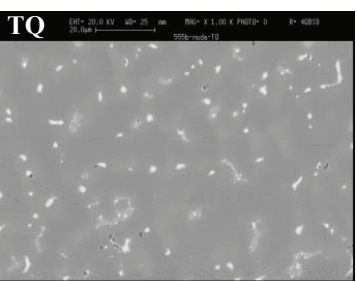

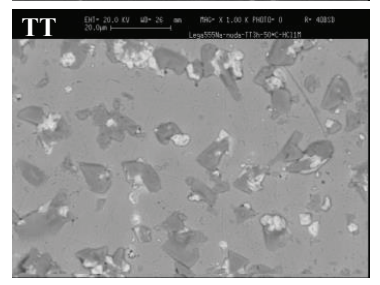

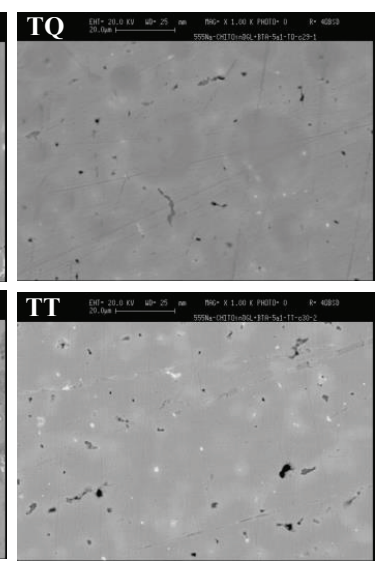

FIGURE 3. Pictures of bare bronze disks on the left and of bronze disks coated with chitosan-BTA film on the right. Comparison between disks before and after corrosion treatments (labelled as TQ and TT, respectively). SEM images of bare disks before and after corrosion treatments are reported.

The pictures of bare and coated disks show that the deposition of a CS film prepared using DGL and containing a small amount of BTA as corrosion inhibitor does not affect the bronze appearance, since the film is colorless and transparent. The corresponding SEM images indicate that the coating is uniform and compact, without any crack, and that the bronze typical features are only slightly covered. To assess the efficacy of the protective polymer layers, accelerated corrosion tests have been carried out on bare and coated bronze disks. SEM images of the alloy surface before and after accelerated corrosion treatments reveal an evident degradation of bare bronze, which is also evident from the corresponding pictures. It is worth noting that, differently from the bare bronze, the appearance of the coated disk after corrosion treatment remain unchanged. To assess if the bronze features were affected by the treatment, the coating was removed from the disk surface after the corrosion test. From the SEM picture in Figure 3, no evidence of corrosion was detected, thus demonstrating the ability of the polymer coating to prevent the degradation of bronze substrates. The obtained results suggest that the coatings are able to provide a safe protection of bronze substrates.

\section{CONCLUSIONS}

The reported activity represents the preliminary results of an ongoing research addressing the preparation of smart coating formulations for the protection of $\mathrm{Cu}$-based alloy artworks stored or exhibited in indoor environment. The obtained results are promising as they suggest that the realized coatings, optically transparent and easily removable, effectively preserve the surface characteristics of bronze substrates during accelerated corrosion tests.

\section{ACKNOWLEDGMENTS}

The activity is carried out within the framework of the NANORESTART project which has received funding from the European Union's Horizon 2020 research and innovation programme under grant agreement No 646063.

\section{REFERENCES}

1. Shchukin DG, Zheludkevich M, Yasakau K, Lamaka S, Ferreira MG, Moehwald H, Adv Mater, 18, 1672-1678, 2006.

2. Xu H, Zhang H, Wang D, Wu L, Liu X, Jiao Z, J Colloid Interf Sci, 451, 101-107, 2015.

3. Shchukin DG, Zheludkevich M, Möhwald H, J Mater Chem, 16, 4561-4566, 2006.

4. Couture-Rigert DE, Jane Sirois P, Moffatt EA, Stud Conserv, 57, 142-163, 2012. 